\title{
Management of ureteral calculi
}

Gerber GS, Acharya SS

Section of Urology, University of Chicago, Chicago, Illinois, USA

J Endourol. 2010; 24: 953-4.

This is the fourth in a series of articles on an electronic survey of practicing urologists in the United States and elsewhere that concerns endourologic management of a variety of conditions. There were 416 e-mail responses to the survey, which was conducted in late 2007 and early 2008. The electronic mail lists of the American Urological Association and the Endourological Society were used. A little more than half of the respondents were from the United States, with 15\% from Europe and 12\% from Asia. Further details regarding the survey can be found in a previous publication. 1

The present report focuses on questions from the survey that concern the treatment of patients with ureteral calculi:

1. Do you have access to the holmium laser for ureteroscopic stone treatment? (Answer choices, yes or no)

2. Which treatment would you recommend for an uncomplicated patient with a symptomatic distal ureteral stone that was 4 (or 8 or 15) mm? (Answer choices, ureteroscopic stone removal, extracorporeal shock wave lithotripsy (SWL) or ureteral stent placement alone)

3. Which treatment would you recommend for an uncomplicated patient with a symptomatic midureteral stone that was 4 (or 8 or 15) mm? (Same answer choices as question 2)

4. Which treatment would you recommend for an uncomplicated patient with a symptomatic proximal ureteral stone that was 4 (or 8 or 15) mm? (Same answer choices as question 2)

\section{Editorial Comment}

The international sample of urologists responding to the questionnaire may not be reflective of the general practicing urologist in those countries - they are selected by their membership in international organizations (AUA) or membership in societies for those with specific interest and/or expertise in endourology (Endourology Society). As such, the survey may overestimate the availability of holmium laser in these countries and the dissemination of advanced endoscopic procedures.

The authors did not stratify responses based on gender of the patient - one might anticipate a higher percentage of urologists may tackle a midureteral or proximal ureteral stone in female than in a male patient due to the ability to access the stone with a semi-rigid ureteroscope.

Interestingly, non-North American urologists were more likely (15\%) than North American urologists (4\%) to stent only for a small $4 \mathrm{~mm}$ stone. This may represent a higher inclination to give the patient an opportunity to pass the stone spontaneously once stented - a two edged sword as it may increase the likelihood for a secondary procedure down the road.

\author{
Dr. Manoj Monga \\ Professor, Department of Urology \\ Cleveland Clinic Foundation \\ Cleveland, Ohio, USA \\ E-mail:endourol@yahoo.com
}

